\title{
Adiabatic connection from accurate wavefunction calculations
}

\author{
Derek Frydel and William H. Terilla \\ Department of Chemistry, Rutgers University, 315 Penn Street, Camden, NJ 08102 \\ Kieron Burke \\ Departments of Chemistry and Physics, Rutgers University, 610 Taylor Road, Piscataway, NJ 08854
}

(Submitted to J. Chem. Phys., Oct. , 1999)

An extremely easy method for accurately calculating the adiabatic connection of density functional theory is presented, and its accuracy tested on both Hooke's atom and the He atom. The method is easy because calculations are needed only for different values of parameters in the external potential, which can be achieved with almost any electronic structure code.

\section{INTRODUCTION}

Density functional theory has become a popular computational method in quantum chemistry, because of its ability to handle large molecules accurately but relatively inexpensively [1,2]. This success is based on the availability of reliable accurate approximate functionals, and there is a constant need for still further accuracy. The goal of atomization energy errors being reliably less than $1 \mathrm{kcal} / \mathrm{mol}$ has not yet been achieved.

An important step forward in this search for accuracy came when Becke mixed a fraction of exact exchange with a generalized gradient approximation (GGA), and reduced errors by a factor of two or three [3]. Such hybrid functionals, e.g., B3LYP, are now in common use, but their underlying justification comes from the adiabatic decomposition of density functional theory. Initially, the mixing parameters used were determined empirically. Later, it was shown that these parameters could be derived non-empirically [A. 5], and that a single universal mixing coefficient $(25 \%)$ could be rationalized based on the performance of MP theory for molecules [6]. Most recently, new functionals have been proposed which use this adiabatic decomposition in much detail [7].

The adiabatic decomposition of an electronic system is very simple conceptually. Imagine multiplying the electron-electron repulsion by a coupling constant $\lambda$. Now imagine varying $\lambda$, while keeping the electron density $\rho(\mathbf{r})$ fixed. This differs from traditional perturbative methods, e.g., Móller-Plesset [8], because the external potential must be altered at each $\lambda$ to keep the density fixed. At $\lambda=1$, we have the physical, interacting electronic system. But as $\lambda$ is reduced to zero, keeping the density fixed, the electrons become those of the non-interacting,
Kohn-Sham system, and the potential morphs into the Kohn-Sham potential. All Kohn-Sham DFT calculations are actually performed on this non-interacting system, and the physical ground-state energy deduced from it. The adiabatic connection provides a continuous connection between the interacting system and its Kohn-Sham analog.

The only part of the total energy which must be approximated in such a Kohn-Sham calculation is the exchange-correlation energy, $E_{\mathrm{xC}}[\rho]$, as a functional of the (spin) density. A further value of the coupling constant is that, through the Hellmann-Feynman theorem, this energy can be written as an intergal over the purely potential contribution [9, 10]:

$$
E_{\mathrm{XC}}=\int_{0}^{1} d \lambda U_{\mathrm{xC}}(\lambda), \quad U_{\mathrm{xC}}(\lambda)=\left\langle\Psi^{\lambda}\left|\hat{V}_{\mathrm{ee}}\right| \Psi^{\lambda}\right\rangle-U,
$$

where $\hat{V}_{\text {ee }}$ is the Coulomb interaction between electrons, $\Psi^{\lambda}$ is the wavefunction at coupling constant $\lambda$, and $U$ is the Hartree electrostatic energy. This integral is the adiabatic connection formula. Hybrid functionals are based on the fact that this integrand, when applied to the exchange-correlation contribution to a bond dissociation energy, is often not well-approximated by GGA's, due to their lack of static correlation [11,12. This can be partially corrected for most molecules by mixing in a fraction of exact exchange. Construction of functionals based on this insight is often referred to as the adiabatic connection method (ACM).

Thus accurate approximation of this adiabatic connection curve is extremely important to further progress in construction of approximate functionals, and benchmark cases for small systems are always of interest and help in this endeavor. However, this is, in principle, a very demanding task. For each value of $\lambda$, one must solve the interacting electronic problem many times in order to find the external potential which reproduces the $\lambda=1$ density. Almbladh and Pedroza 13 made early heroic attempts, but not with the accuracy of modern calculations. In the last several years, as the importance of these curves has become apparent, several groups in different areas have performed adiabatic decomposition calculations. Hood et al. 14 have calculated the curve for bulk Si. Colonna and Savin [15] have used the slightly different Harris and Jones 16] decomposition for both the $\mathrm{He}$ and Be isoelectronic series. Joubert and Srivastava 17 


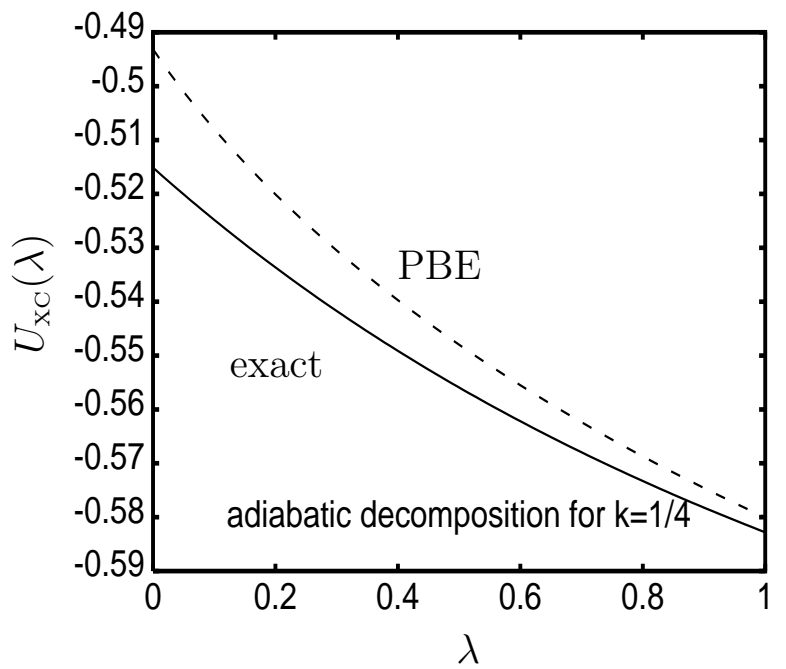

FIG. 1. Exact $U_{\mathrm{XC}}(\lambda)$ curve for the $\mathrm{k}=1 / 4$ Hooke's atom, (solid line) and within the PBE correlation functional (dashed line), calculated as described in the text (atomic units).

have used Hylleras-type wavefunctions to calculate these curves for the He isoelectronic series.

All these methods require solving the interacting electronic problem with a potential that differs from the original external potential, e.g., $-Z / r$ for atoms. This typically makes them difficult to transfer to other systems, radically different from the original one, in which other codes and approximations are being used. In this paper, we show how to construct the adiabatic connection formula for any atom accurately by doing calculations simply for different values of $Z$, so that no modification need be made to an existing wavefunction code. A standard calculation is simply run for several different nuclear charges, running from the physical value up to $Z=\infty$. The main focus of this paper is to demonstrate the accuracy of the method. The solid line in Figure 1 is an essentially exact curve for $U_{\mathrm{xC}}(\lambda)$ for Hooke's atom (two electrons in a harmonic potential), for a spring constant of $k=1 / 4$ (atomic units), calculated from a series of exact calculations for force constants greater than $1 / 4$, but never requiring any calculation with a different external potential. The method can be immediately applied to accurate calculations for larger atoms, and is currently being explored for molecules.

The paper is divided into several sections. In the next section, we outline the basic theory behind our calculations. Following that, we present results, both using the exact functional, and within a GGA, for both Hooke's atom and the He atom. Hooke's atom consists of two electrons attached by springs to a center, but interacting via a Coulomb repulsion. We summarize our findings in the last section. Atomic units $\left(e^{2}=\hbar=m_{e}=1\right)$ are used throughout, and only spin-unpolarized systems are discussed.

\section{THEORY}

\section{A. Formally exact results}

For convenience, we begin with a review of the adiabatic connection formalism. We define $\Psi^{\lambda}[\rho]$ to be that wavefunction which has density $\rho(\mathbf{r})$ and minimizes

$$
F^{\lambda}[\Psi]=\left\langle\Psi\left|\hat{T}+\lambda \hat{V}_{\mathrm{ee}}\right| \Psi\right\rangle .
$$

Then the kinetic-correlation energy at $\lambda$ is

$$
T_{\mathrm{C}}^{\lambda}=\left\langle\Psi^{\lambda}|\hat{T}| \Psi^{\lambda}\right\rangle-T_{\mathrm{S}}[\rho]
$$

where $T_{\mathrm{S}}[\rho]$ is the non-interacting Kohn-Sham kinetic energy of density $\rho$. The potential contribution to exchange-correlation at coupling constant $\lambda$ is,

$$
U_{\mathrm{xC}}^{\lambda}=\lambda\left\langle\Psi^{\lambda}\left|\hat{V}_{\mathrm{ee}}\right| \Psi^{\lambda}\right\rangle-\lambda U[\rho]
$$

while the exchange-correlation energy at $\lambda$ is

$$
E_{\mathrm{xC}}^{\lambda}=U_{\mathrm{xC}}^{\lambda}+T_{\mathrm{C}}^{\lambda} \text {. }
$$

For $\lambda=1$, the system becomes the true interacting quantum-mechanical system, and energies without superscripts refer to $\lambda=1$, e.g., $E_{\mathrm{XC}}=E_{\mathrm{XC}}^{\lambda=1}$.

All quantities at coupling constants different from one are simply related to their full coupling strength counterparts, but evaluated on a scaled density [18]. The most important examples are the wavefunction:

$$
\Psi^{\lambda}[\rho]=\Psi_{\lambda}\left[\rho_{1 / \lambda}\right]
$$

and the exchange-correlation energy

$$
E_{\mathrm{XC}}^{\lambda}[\rho]=\lambda^{2} E_{\mathrm{XC}}\left[\rho_{1 / \lambda}\right]
$$

where

$$
\rho_{\gamma}(\mathbf{r}) \quad=
$$
and $\Psi_{\gamma}\left(\mathbf{r}_{1} . . \mathbf{r}_{N}\right)=\gamma^{3 / 2} \Psi_{\gamma}\left(\gamma \mathbf{r}_{1 . .} \gamma \mathbf{r}_{N}\right)$. Thus knowledge of how a quantity varies as the density is scaled implies knowledge of its coupling constant dependence.

Quantities evaluated on the Kohn-Sham wavefunction vary in a simple way, such as the non-interacting kinetic energy $T_{\mathrm{s}}$ :

$$
T_{\mathrm{S}}^{\lambda}[\rho]=T_{\mathrm{S}}[\rho], \quad \text { or } \quad T_{\mathrm{S}}\left[\rho_{\gamma}\right]=\gamma^{2} T_{\mathrm{S}}[\rho]
$$

the Hartree electrostatic energy,

$$
U^{\lambda}[\rho]=\lambda U[\rho], \quad \text { or } \quad U\left[\rho_{\gamma}\right]=\gamma U[\rho],
$$

and the exchange energy

$$
E_{\mathrm{x}}^{\lambda}[\rho]=\lambda E_{\mathrm{x}}[\rho], \quad \text { or } \quad E_{\mathrm{X}}\left[\rho_{\gamma}\right]=\gamma E_{\mathrm{x}}[\rho] .
$$

Correlation is more sophisticated. Note, however, that knowledge of any quantity, $E_{\mathrm{C}}\left[\rho_{\gamma}\right], T_{\mathrm{C}}\left[\rho_{\gamma}\right]$, or $U_{\mathrm{C}}\left[\rho_{\gamma}\right]$ as a function of $\gamma$ for $\gamma$ between 1 and $\infty$, i.e., scaling to 
the high density limit, is sufficient to determine the adiabatic connection for any of them, for $\lambda$ between 0 and 1 . The most well-known relation is to extract the kineticcorrelation piece from $E_{\mathrm{C}}[\rho]$ [18]:

$$
T_{\mathrm{C}}[\rho]=-E_{\mathrm{C}}[\rho]+\left.\frac{d E_{\mathrm{C}}\left[\rho_{\gamma}\right]}{d \gamma}\right|_{\gamma=1} .
$$

To generalize this result to $\rho_{\gamma}$, apply Eq. (11) to $\rho_{\gamma}$, and make a change of variables in the derivative, to find:

$$
T_{\mathrm{C}}\left[\rho_{\gamma}\right]=-E_{\mathrm{C}}\left[\rho_{\gamma}\right]+\gamma \frac{d E_{\mathrm{C}}\left[\rho_{\gamma}\right]}{d \gamma}
$$

This can be considered a first-order differential equation in $\gamma$ for $E_{\mathrm{C}}\left[\rho_{\gamma}\right]$. Solution of this equation yields

$$
E_{\mathrm{C}}\left[\rho_{\gamma}\right]=-\gamma \int_{\gamma}^{\infty} \frac{d \gamma^{\prime}}{\gamma^{\prime 2}} T_{\mathrm{C}}\left[\rho_{\gamma^{\prime}}\right]
$$

where we have used the fact that $E_{\mathrm{C}}\left[\rho_{\gamma}\right]$ has vanished as $\gamma \rightarrow 0$ [19. Via Eq. (7), these can be turned into coupling-constant relations:

$$
T_{\mathrm{C}}^{\lambda}=E_{\mathrm{C}}^{\lambda}-\lambda \frac{d E_{\mathrm{C}}^{\lambda}}{d \lambda},
$$

and

$$
E_{\mathrm{C}}^{\lambda}=-\lambda \int_{0}^{\lambda} \frac{d \lambda^{\prime}}{\lambda^{\prime 2}} T_{\mathrm{C}}^{\lambda^{\prime}} .
$$

The latter is called Bass' relation 20]. Similarly, we can extract $U_{\mathrm{C}}$ from $E_{\mathrm{C}}\left[\rho_{\gamma}\right]$, since $U_{\mathrm{C}}=E_{\mathrm{C}}-T_{\mathrm{C}}$. Thus Eq. (12) leads to

$$
U_{\mathrm{C}}\left[\rho_{\gamma}\right]=2 E_{\mathrm{C}}\left[\rho_{\gamma}\right]-\gamma \frac{d E_{\mathrm{C}}\left[\rho_{\gamma}\right]}{d \gamma}
$$

which, inverted, is

$$
E_{\mathrm{C}}\left[\rho_{\gamma}\right]=\gamma^{2} \int_{\gamma}^{\infty} \frac{d \gamma^{\prime}}{\gamma^{\prime 3}} U_{\mathrm{C}}\left[\rho_{\gamma^{\prime}}\right] .
$$

Combining Eqs. (16) and (13) then leads to simple relations between $U_{\mathrm{C}}$ and $T_{\mathrm{C}}$. Lastly, the coupling-constant relation that follows from Eq. (17) is

$$
E_{\mathrm{C}}[\rho]=\int_{0}^{1} \frac{d \lambda}{\lambda} U_{\mathrm{C}}^{\lambda}[\rho],
$$

and $U_{\mathrm{xC}}(\lambda)$ of Fig. 1 is just the integrand when this expression is applied to both exchange and correlation, i.e., $U_{\mathrm{xC}}^{\lambda} / \lambda$. We emphasize that all these relations follow from the well-known Eqs. (『) and (11).

\section{B. Highly accurate approximations}

We begin this section by noting how, when some parameter in an external potential is altered, the density changes scale, but often does not change shape very much. For example, for the two-electron ion, going from $\mathrm{Z}=2$ to $\mathrm{Z}=4$ will roughly multiply the density by 8 , and reduce its length scale by a factor of 2 . We use this fact to very accurately approximate scaling the density. Then, through the relations derived above, we can convert this into the coupling-constant dependence.

Let $\rho(\mathbf{r})$ be the density of the system we are interested in. Suppose we alter some parameter in the external potential, and solve the interacting problem, finding some density $\rho^{\prime}(\mathbf{r})$. If we want to treat $\rho^{\prime}$ as an approximation to $\rho_{\gamma}$, we must first choose a criterion for determining $\gamma$. In fact, either of Eqs. (8) and (9) could be used, since both would be satisfied exactly if $\rho^{\prime}$ were truly a scaled density. For definiteness, we choose

$$
\lambda=1 / \gamma=U[\rho] / U\left[\rho^{\prime}\right]
$$

We can then consider $E_{\mathrm{C}}\left[\rho^{\prime}\right] \approx E_{\mathrm{C}}\left[\rho_{\gamma}\right], T_{\mathrm{C}}\left[\rho^{\prime}\right] \approx T_{\mathrm{C}}\left[\rho_{\gamma}\right]$, etc., which we call the bare estimates. As we shall show in the results section, these energies usually yield quite accurate approximations to the exact quantities. However, since we are not working with the true scaled density, these energies do not satisfy the relations involving scaling derivatives above, such as Eq. (13).

To make a better estimate, we note that, in the case of the correlation energy, we can calculate the leading correction to $E_{\mathrm{C}}\left[\rho_{\gamma}\right]$, using the correlation potential. This can be constructed by finding the exact Kohn-Sham potential which generates the density, and subtracting from it the external, Hartree, and exchange contributions. Then,

$$
E_{\mathrm{C}}\left[\rho_{\gamma}\right] \approx E_{\mathrm{C}}\left[\rho^{\prime}\right]+\int d^{3} r v_{\mathrm{C}}\left[\rho^{\prime}\right](\mathbf{r})\left(\rho_{\gamma}(\mathbf{r})-\rho^{\prime}(\mathbf{r})\right)+O(\delta \rho)^{2} .
$$

As will be shown below, inclusion of this correction leads to extremely accurate adiabatic connection curves. We generate $T_{\mathrm{C}}\left[\rho_{\gamma}\right]$ using Eq. (13), and then $U_{\mathrm{C}}=E_{\mathrm{C}}-T_{\mathrm{C}}$. In particular, as $\gamma \rightarrow 1, \rho^{\prime} \rightarrow \rho$, and Eq. (20) becomes exact to first order in the difference between $\rho^{\prime}$ and $\rho$. Thus Eq. (11) is satisfied exactly.

Lastly, we apply the same principles to $T_{\mathrm{S}}$, as a test of the closeness of the approximate density, $\rho^{\prime}$. The Euler equation for the Kohn-Sham system says that

$$
\frac{\delta T_{\mathrm{S}}}{\delta \rho(\mathbf{r})}=\mu-v_{\mathrm{S}}(\mathbf{r})
$$

where $\mu$ is a constant, and $v_{\mathrm{S}}(\mathbf{r})$ is the Kohn-Sham potential. Thus, we can also correct the bare $T_{\mathrm{S}}$ estimate, to 
$T_{\mathrm{S}}\left[\rho_{\gamma}\right]=T_{\mathrm{S}}\left[\rho^{\prime}\right]-\int d^{3} r v_{\mathrm{S}}\left[\rho^{\prime}\right](\mathbf{r})\left(\rho_{\gamma}(\mathbf{r})-\rho^{\prime}(\mathbf{r})\right)+O(\delta \rho)^{2}$.

\section{RESULTS}

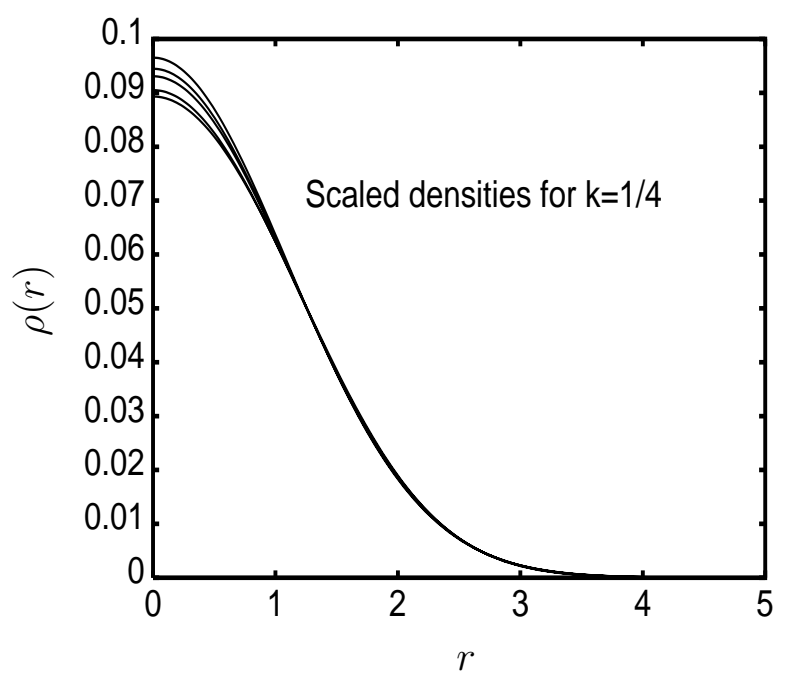

FIG. 2. Densities used for $\lambda=1$ (least $\rho(0)$ ), 0.75, 0.5, 0.25 , and 0 (largest $\rho(0))$.

We present results for two systems, Hooke's atom and the He atom. The first of these contains two electrons in a harmonic potential, interacting via a Coulomb repulsion. This provides a valid test case for density functional theory, because of the Coulomb repulsion between electrons. It is also an easy system to perform calculations on, because the center-of-mass and relative coordinates separate, leaving only a one-dimension differential equation to solve numerically [21]. Even this equation has an analytic solution for force constant $k=1 / 4$ [22], which is the system on which we will demonstrate most of our results.

To implement our method for the $k=1 / 4$ Hooke's atom, we run many calculations at different values of $k>1 / 4$, up to $k=10^{6}$, using the method of Ref. [21. For each calculation, we scale the density so that it looks like the original $k=1 / 4$ density, using Eq. (19) to define the scale factor. Several such densities are shown in Fig. 2 , which illustrates how close these densities are. Note that the largest error is for $\lambda=0$, the non-interacting Gaussian density.

Figure 3 is a plot of the three quantities $E_{\mathrm{C}}\left[\rho_{1 / \lambda}\right]$, $T_{\mathrm{C}}\left[\rho_{1 / \lambda}\right]$, and $U_{\mathrm{C}}\left[\rho_{1 / \lambda}\right]$, all found using the PBE correlation functional. We choose these quantities to plot, as they remain finite in the range $\lambda=0$ to 1 . For each quantity, there are two curves. The dashed curve is the bare result of the calculations at different values of the force constant, i.e., using $E\left[\rho^{\prime}\right]$ alone. The solid curve

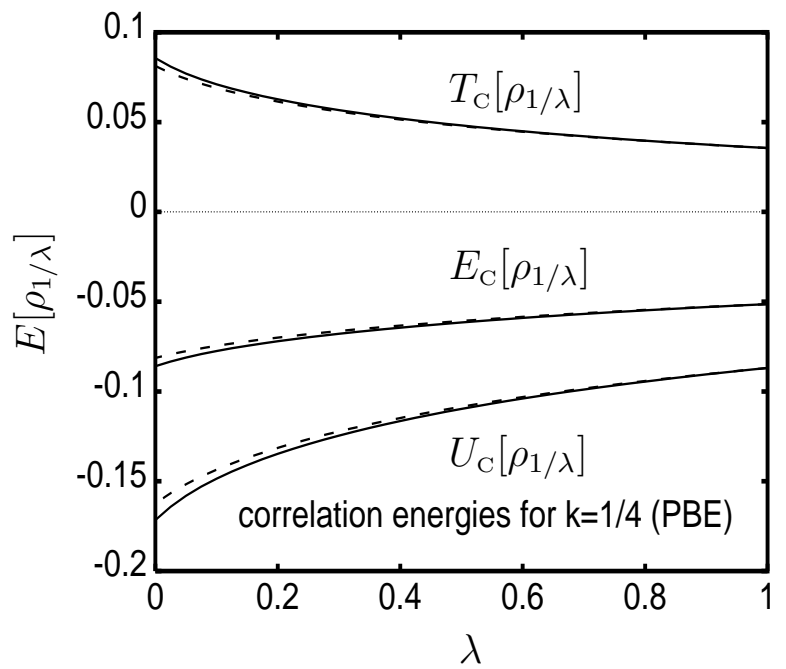

FIG. 3. Correlation energies for the $\mathrm{k}=1 / 4$ Hooke's atom, calculated both with (solid lines) and without (dashed lines) the correction term, using the PBE correlation functional. The solid lines are indistinguishable from exact results, calculated by scaling the PBE correlation functional.

for $E_{\mathrm{C}}\left[\rho_{1 / \gamma}\right]$ includes the correction due to the potential, in accordance with Eq. (20). The $U_{\mathrm{C}}$ and $T_{\mathrm{C}}$ curves are then extracted from this one, using Eqs. (12) and (16). Both the value and first derivative at $\lambda=1$ are exact for this curve. What is more remarkable is that these curves coincide essentially exactly with the correct result, which we can deduce by directly applying Eq. (7) to the PBE correlation energy functional. Careful numerical calculations indicate that the maximum error in our curve is at $\lambda=0$, and is less than $3 \times 10^{-4}$ Hartrees. This is due to the similarity of densities, as shown in Fig. 2, leading to very small corrections.

We also tested other possible prescriptions for choosing $\lambda$, such as from the square root of the ratio of noninterating kinetic energy densities, as in Eq. (8). This gives values for $\lambda$ very close to those of Eq. (9), and leads to no measurable change in our estimate for $E_{\mathrm{C}}\left[\rho_{1 / \lambda}\right]$.

Figure 4 is the analog of Fig. 3, but now calculated using exact correlation energies and potentials. Based on the remarkable accuracy of Fig. 3, we claim these curves are essentially exact. In fact, the change due to the potential correction is much smaller for the exact case than for PBE, suggesting that the error made in Eq. (20) will also be smaller. The adiabatic curve of Fig. 1 was derived from these curves, since $U_{\mathrm{xC}}(\lambda)=\lambda U_{\mathrm{xC}}\left[\rho_{1 / \lambda}\right]$.

We also ran PBE calculations for lower densities, where the shape of the density can change significantly with $k$. We find that even as low as $k=10^{-4}$, there is a maximum error of only 1 millihartree in the corrected $E_{\mathrm{C}}\left[\rho_{1 / \lambda}\right]$ curve. Beyond this point, the reliability of our method might be questioned. However, defining an average $r_{s}$ value via Eq. (6a) of Ref. [23], at this point $\left\langle r_{s}\right\rangle=19$, whereas for $k=1 / 4,\left\langle r_{s}\right\rangle$ is 2.07 . Thus for common val- 


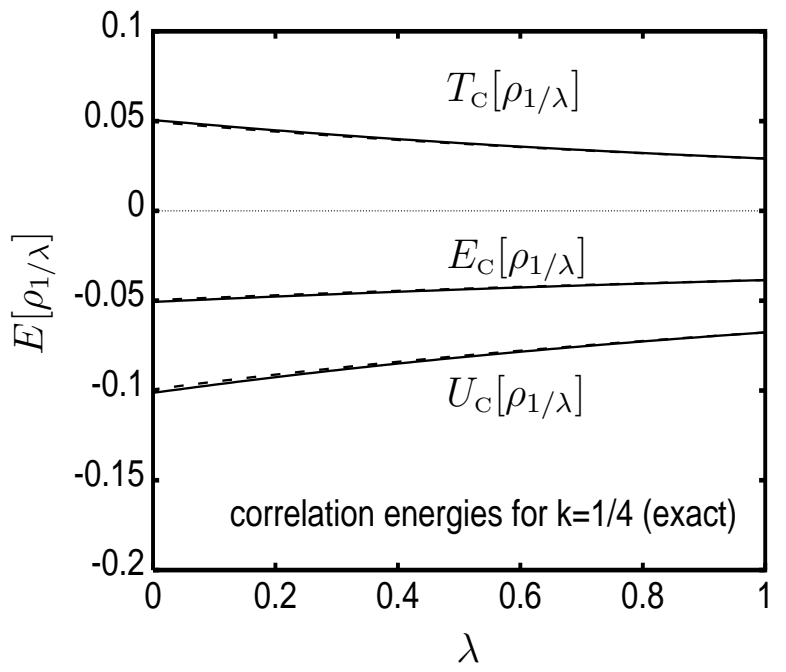

FIG. 4. Correlation energies for the $k=1 / 4$ Hooke's atom, calculated both with (solid lines) and without (dashed lines) the correction term.

ues of the density, the errors in our procedure are minute.

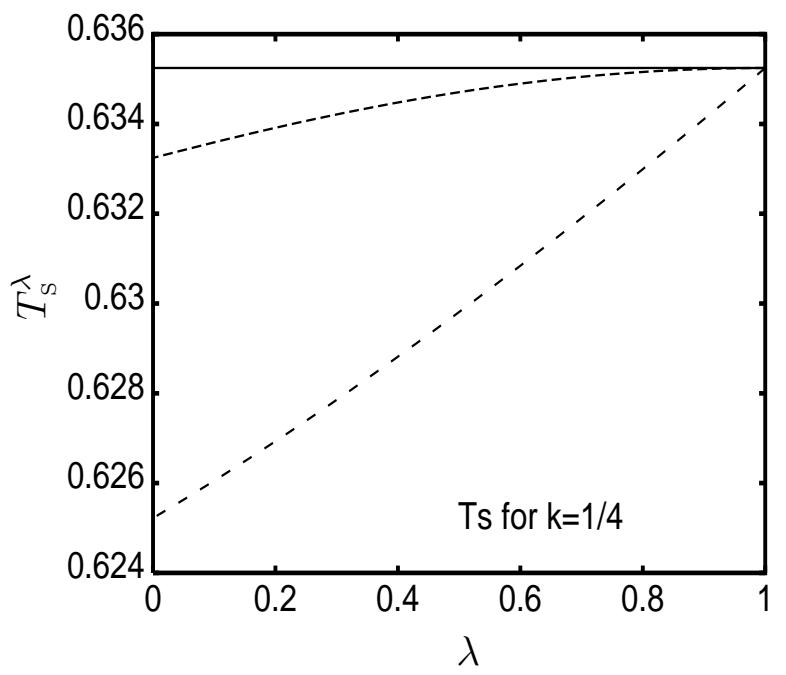

FIG. 5. Non-interacting kinetic energy as a function of coupling constant. Exact quantity (solid line) is independent of $\lambda$. Dashed line is bare estimate of $T_{\mathrm{S}}$, the dotted line includes the correction.

As a final test of our method, we return to the noninteracting kinetic energy. If the scaling were exact, the non-interacting kinetic energy would scale quadratically, as in Eq. (8). In Figure 5, we plot $T_{\mathrm{S}}\left[\rho_{\gamma}\right] / \gamma^{2}$ exactly, approximating $T_{\mathrm{S}}\left[\rho_{\gamma}\right]$ by $T_{\mathrm{S}}\left[\rho^{\prime}\right]$, and including the correction of Eq. (22). Note that the maximum absolute error, at $\lambda=0$, is only about $-1.6 \%$, in the bare estimate. The correction makes the derivative with respect to $\lambda$ exact as $\lambda \rightarrow 1$, and overall reduces the error by about a factor of 5 , to about $-0.3 \%$.

Finally, we discuss our results for the two-electron ion series, simply to demonstrate that there is nothing special about Hooke's atom which makes these techniques

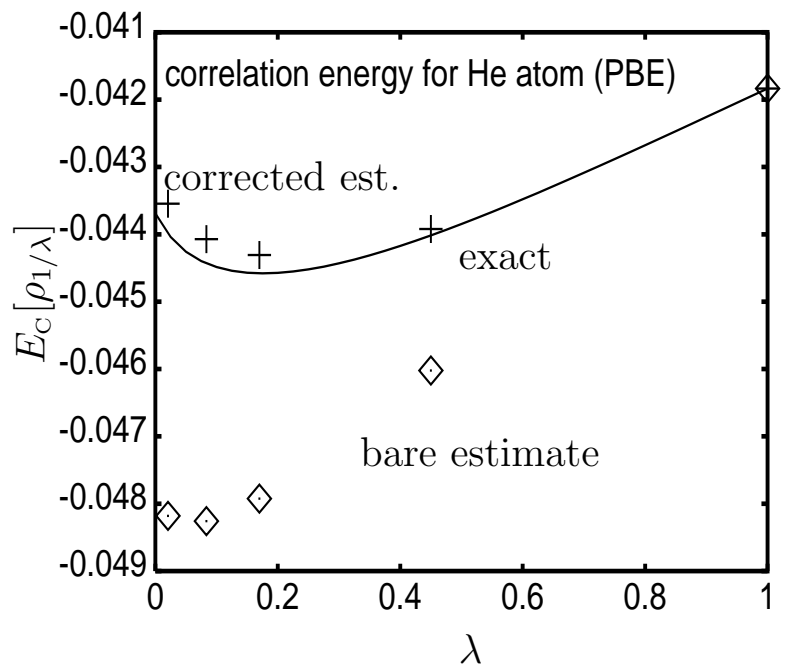

FIG. 6. Correlation energy for the He atom, calculated both without (diamonds) and with (crosses) the correction term, using the PBE correlation functional. The solid line is the exact result, calculated by scaling the $\mathrm{PBE}$ correlation functional.

accurate. We may make bare estimates of the adiabatic curves directly from energy data already in the literature. Thus by calculating $\lambda$ by Eq. (19) using the data in Table I of Ref. [24], and assigning to each $\lambda$ the bare $U_{\mathrm{C}}$ value from the table, we find the corresponding adiabatic connection curve integrates to $E_{\mathrm{C}}=-41.5 \mathrm{mH}$, as compared with an exact value of $-42.1 \mathrm{mH}$ for He. We can similarly use the $T_{\mathrm{C}}$ data in Eq. (13) to get an estimate of -41.2 $\mathrm{mH}$, so that the difference between these two results is a good indicator of the error in both of them. Again, we can calculate $T_{\mathrm{S}}^{\lambda}$, and find the largest error at $\lambda=0$, with a value of 2.916 , as compared to the exact value of 2.867 .

In Fig. 6, we plot PBE calculations of $E_{\mathrm{C}}\left[\rho_{1 / \lambda}\right]$ for the He atom, both exactly and using the fake adiabatic connection formula, both with and without the correction term. We use the highly accurate densities of Umrigar and Gonze [25]. Unfortunately, not enough data points are available to make smooth plots for this system. Already at $Z=3, \lambda=0.62$, so that results for non-integer values of $Z$ (between $Z=2$ and 3) are needed. However, enough data is present to see the clear correction the potential makes. We note several interesting features of this curve. First, the dependence on scaling is much less for He than for the $k=1 / 4$ Hooke's atom. Second, the PBE curve contains a minimum, so Eq. 12 implies $E_{\mathrm{C}}+T_{\mathrm{C}}$ becomes positive for this scaled density. While it has never been rigorously proven, every known case of $E_{\mathrm{C}}+T_{\mathrm{C}}$ is negative [18,26]. Thus this is probably a (very slight) limitation of PBE, which does not occur in PW91, and may be related to the difference at $r_{s}=0$ in Fig. 7 of Ref. [27]. Third, the potential correction even picks up this feature, and still only has errors of a fraction of 
a millihartree.

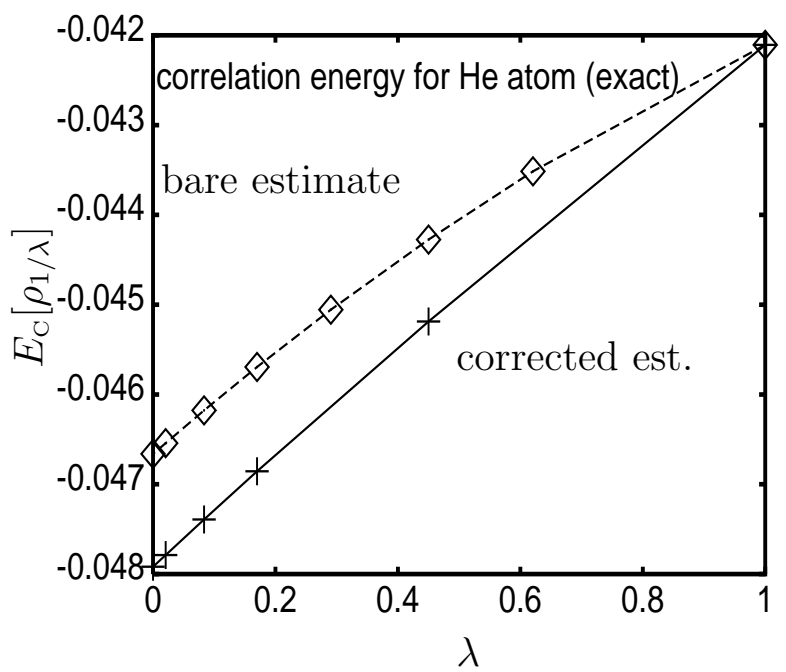

FIG. 7. Correlation energy for the He atom, calculated both without (diamonds) and with (crosses) the correction term.

Figure 7 repeats Fig. 6, but for the exact case. Here the corrected curve is much straighter than for PBE, and has no minimum. The lines are drawn merely to aid the eye. Note that the value of $E_{\mathrm{C}}^{(2)}[\rho]$, which is the highdensity limit of $E_{\mathrm{C}}$, drops about 1 millihartree, due to the difference in shape between the He atom and a pure exponential decay (bare estimate). We find $E_{\mathrm{C}}^{(2)}=-47.9$ millihartree, in reasonable agreement with the value of Colonna and Savin [15] (-47.5 from Table VI), of Joubert and Srivastava [17] (-47.2 from $a_{p}$ in Table II), and of Engel and Dreizler [28] (-48.2 from Table V). The value -50.3 , reported in Ref. [29], was evaluated on the PBE selfconsistent density.

\section{CONCLUSIONS AND IMPLICATIONS}

We have shown how, with accurate ground-state results as a function of the external potential, an accurate adiabatic connection curve can be calculated for both Hooke's atom at $k=1 / 4$ and the He atom. We see no reason why similar results could not be obtained for larger atoms, especially those for which the Kohn-Sham potential has already been calculated [30]. There also exist methods for isolating the correlation potential [31.

In the event that the exchange contribution cannot be easily isolated, the scheme can still be applied, but now to the combined exchange-correlation energy at each value of the external parameter. The correction is now evaluated using the exchange-correlation potential. On Hooke's atom, this yields results almost as accurate as those described for the correlation energy alone. Important differences are in the regime $\lambda \rightarrow 0$, as here the exchange contribution to the correction blows up, since $\gamma=1 / \lambda \rightarrow \infty$. This effect causes noticeable errors only for $\lambda<0.2$ in $E_{\mathrm{C}}\left[\rho_{1 / \lambda}\right]$, but not in $U_{\mathrm{C}}$ or $T_{\mathrm{C}}$. It also means that neither $U_{\mathrm{C}}[\rho]$ or $T_{\mathrm{C}}[\rho]$, as derived from the resulting $E_{\mathrm{C}}\left[\rho_{\gamma}\right]$ curve via Eqs. (16) and (12), is exact at $\lambda=1$. However, these differences are of the order of 0.1 millihartree.

Finally, we are currently investigating if this method can be used to calculate accurate adiabatic connection curves for molecules [12].

\section{ACKNOWLEDGMENTS}

This work was supported by an award from Research Corporation and by the National Science Foundation under grant No. CHE-9875091. We thank John Perdew for his comments, and Cyrus Umrigar for providing us with accurate densities for the two electron ions.

[1] Density functional calculations of planar DNA base-pairs, M. Machado, P. Ordejón, E. Artacho, D. Sánchez-Portal, and José M. Soler, physics preprint 9908022.

[2] Density functional theory calculations of the visible spectrum of chlorophyll a, D. Sundholm, Chem. Phys. Lett., 302, 480 (1999).

[3] Density-functional thermochemistry. III. The role of exact exchange, A.D. Becke, J. Chem. Phys. 98, 5648 (1993).

[4] The adiabatic connection method: A non-empirical hybrid, K. Burke, M. Ernzerhof, and J.P. Perdew, Chem. Phys. Lett. 265, 115 (1997).

[5] M. Ernzerhof, Chem. Phys. Lett. 263, 499 (1996).

[6] Rationale for mixing exact exchange with density functional approximations, J.P. Perdew, M. Ernzerhof, and K. Burke, J. Chem. Phys. 105, 9982 (1996).

[7] Divergence and accurate re-summation of densityfunctional perturbation theory: Making correlation compatible with exact exchange, M. Seidl, J.P. Perdew, and S. Kurth, preprint, Aug. 1999.

[8] C. Møller and M. S. Plesset, Phys. Rev. 46, 618 (1934).

[9] The exchange-correlation energy of a metallic surface, D.C. Langreth and J.P. Perdew, Solid State Commun. 17, 1425 (1975).

[10] O. Gunnarsson and B.I. Lundqvist, Phys. Rev. B 13, 4274 (1976).

[11] Fermi holes and Coulomb holes, M.A. Buijse and E.J. Baerends, in Density Functional Theory of Molecules, Clusters, and Solids, ed. D.E. Ellis (Kluwer Academic Publishers, Amsterdam, 1995).

[12] Coupling-constant dependence of atomization energies, M. Ernzerhof, J.P. Perdew, and K. Burke, Int. J. Quantum Chem. 64, 285 (1997).

[13] Density-functional exchange-correlation potentials and 
orbital eigenvalues for light atoms, C.-O. Almbladh and A.C. Pedroza, Phys. Rev. A 29, 2322 (1984).

[14] Exchange and correlation in silicon, R.Q. Hood, M.Y. Chou, A.J. Williamson, G. Rajagopal, and R.J. Needs, Phys. Rev. B 57, 8972 (1998).

[15] Correlation energies for some two- and four-elactron systems along the adiabatic connection in density functional theory, F. Colonna, A. Savin, J. Chem. Phys. 110, 2828 (1999).

[16] J. Harris and R.O. Jones, J. Phys. F 4, 1170 (1974).

[17] Coupling-constant dependence of the density functional correlation energy, D.P. Joubert and G.P. Srivastava, J. Chem. Phys. 109, 5212 (1998).

[18] Hellmann-Feynman, virial, and scaling requisites for the exact universal density functionals. Shape of the correlation potential and diamagnetic susceptibility for atoms, M. Levy and J.P. Perdew, Phys. Rev. A 32, 2010 (1985).

[19] Density-functional exchange-correlation through coordinate scaling in adiabatic connection and correlation hole, M. Levy, Phys. Rev. A 43, 4637 (1991).

[20] Kinetic energy of an electron gas, R. Bass, Phys. Rev. B 32, 2670 (1985).

[21] Exact high-density limit of correlation potential for twoelectron density, S. Ivanov, K. Burke, and M. Levy, J. Chem. Phys. 110, 10262 (1999).

[22] Two electrons in an external oscillator potential: Particular analytic solutions of a Coulomb correlation problem, M. Taut, Phys. Rev. A 48, 3561 (1993).

[23] Distributions and averages of electron density parameters: Explaining the effects of gradient corrections, A. Zupan, K. Burke, M. Ernzerhof, and J.P. Perdew, J. Chem. Phys. 106, 10184 (1997).

[24] Local correlation energies of two-electron atoms and model systems, C.J. Huang and C.J. Umrigar, Phys. Rev. A 56, 290 (1997).

[25] Accurate exchange-correlation potentials and total-energy components for the helium isoelectronic series, C. J. Umrigar and X. Gonze, Phys. Rev. A 50, 3827 (1994).

[26] Digging into the exchange-correlation energy: The exchange-correlation hole, K. Burke in Electronic Density Functional Theory: Recent Progress and New Directions, eds. J.F. Dobson, G. Vignale, and M.P. Das (Plenum, NY, 1997), page 19.

[27] Generalized gradient approximation for the exchangecorrelation hole of a many-electron system, J.P. Perdew, K. Burke, and Y. Wang, Phys. Rev. B 54, 16533 (1996); 57, 14999 (1998) (E).

[28] From explicit to implicit density functionals, E. Engel and R.M. Dreizler, J. Comput. Chem. 20, 31 (1998).

[29] Strictly correlated electrons in density-functional theory, M. Seidl, J.P. Perdew, and M. Levy, Phys. Rev. A 59, 51 (1999).

[30] From electron densities to Kohn-Sham kinetic energies, orbital energies, exchange-correlation potentials, and exchange-correlation energies, Q. Zhao, R.C. Morrison, and R.G. Parr, Phys. Rev. A 50, 2138 (1994).

[31] Separation of the exchange-correlation potential into exchange plus correlation: An optimized effective potential approach, C. Filippi, C. Umrigar, and X. Gonze, Phys. Rev. A 54, 4810 (1996). 